\title{
Enhanced di-Higgs boson production in the complex Higgs singlet model
}

\author{
S. Dawson ${ }^{1}$ and M. Sullivan ${ }^{1,2}$ \\ ${ }^{1}$ Department of Physics, Brookhaven National Laboratory, Upton, New York 11973, USA \\ ${ }^{2}$ Department of Physics and Astronomy, University of Kansas, Lawrence, Kansas 66045, USA
}

(Received 24 November 2017; published 31 January 2018)

\begin{abstract}
We consider the standard model (SM) extended by the addition of a complex scalar singlet, with no assumptions about additional symmetries of the potential. This model provides for resonant di-Higgs production of Higgs particles with different masses. We demonstrate that regions of parameter space allowed by precision electroweak measurements, experimental limits on single Higgs production, and perturbative unitarity allow for large di-Higgs production rates relative to the SM rates. In this scenario, the dominant production mechanism of the new scalar states is di-Higgs production. Results are presented for $\sqrt{S}=13,27$ and $100 \mathrm{TeV}$.
\end{abstract}

DOI: 10.1103/PhysRevD.97.015022

\section{INTRODUCTION}

The exploration of the Higgs sector is a primary focus of the LHC physics program, with measurements of the Higgs couplings to fermions and gauge bosons, the Higgs mass, and Higgs $C P$ properties becoming ever more precise. Very little is known, however, about the Higgs tri-linear and quartic self-couplings which are unambiguously predicted in the standard model (SM). The SM Higgs trilinear coupling can be most sensitively probed by double Higgs production through gluon fusion which unfortunately has a very small rate [1], even at high energy and high luminosity [2]. The best current limit on double Higgs production is from the ATLAS experiment [3], $\sigma(p p \rightarrow h h) / \sigma(p p \rightarrow h h)_{\mathrm{SM}}<29$, with prospects for only modest improvements at higher luminosity. A definitive measurement of the SM tri-linear Higgs self-coupling appears out of reach at the LHC [4-6].

Given the small SM rate for double Higgs production, it is an excellent place to search for beyond the SM (BSM) physics. In the presence of a scalar resonance coupling to the SM-like Higgs boson, the double Higgs rate can be significantly enhanced. This can occur in the minimal supersymmetric standard model (MSSM) and the next-tominimal supersymmetric standard model (NMSSM), for example. The simplest possibility is to add a hypercharge- 0 real scalar to the model which interacts with SM fermions and gauge bosons only through the mixing with the Higgs doublet. The LHC phenomenology in the context of the real

Published by the American Physical Society under the terms of the Creative Commons Attribution 4.0 International license. Further distribution of this work must maintain attribution to the author(s) and the published article's title, journal citation, and DOI. Funded by SCOAP. singlet model has been extensively studied in the literature [7-14]. When the most general scalar potential (without the imposition of a $Z_{2}$ symmetry) is considered, the real singlet model can have a first order electroweak phase transition [15-18] for some values of the parameters.

The complex scalar singlet extension has new features beyond the real singlet case. It has several phases, 2 of which can accommodate a dark matter candidate $[19,20]$. In the broken phase of this model (which is the subject of this work) there are 3 neutral scalar particles which mix to form the mass eigenstates, one of which is the $125 \mathrm{GeV}$ scalar. Final states with 2 different mass scalar particles can be resonantly produced in this scenario and there are large regions of parameter space where the couplings of the new scalars to SM particles are highly suppressed, making the dominant production mechanism of the new scalars the Higgs decays to other Higgs-like particles. The resonant production of two different mass Higgs particles is a smoking gun for this class of theories.

We study the most general case of a complex scalar singlet extension of the SM, without the introduction of any new symmetries for the potential. The complex singlet model has been previously studied imposing a softly broken $U(1)$ symmetry and benchmark points described for the study of the decay of the heavy scalar to the SM Higgs boson and the lighter scalar of the model [21,22]. The parameter space of the model we study is larger, allowing for new phenomenology. The basic features of the model are discussed in Sec. II and the limits on the model from perturbativity, unitarity and the oblique parameters are presented in Sec. III. Our most interesting results are the implications for double Higgs studies and the description of scenarios where one of the new Higgs bosons is predominantly produced in association with the $125 \mathrm{GeV}$ boson. This is discussed in Sec. IV. 


\section{MODEL}

We consider a model containing the SM $S U(2)$ doublet, $\Phi$, and a complex scalar singlet, $S_{c}$. Since $S_{c}$ has hypercharge - 0 it does not couple directly to SM fermion or gauge fields, and its tree level interactions with SM fermions and gauge bosons result entirely from mixing with $\Phi$. The most general renormalizable scalar potential is [23],

$$
\begin{aligned}
\mathcal{V}\left(\Phi, S_{c}\right)= & \frac{\mu^{2}}{2} \Phi^{\dagger} \Phi+\frac{\lambda}{4}\left(\Phi^{\dagger} \Phi\right)^{2} \\
& +\left(\frac{1}{4} \delta_{1} \Phi^{\dagger} \Phi S_{c}+\frac{1}{4} \delta_{3} \Phi^{\dagger} \Phi S_{c}^{2}+a_{1} S_{c}\right. \\
& +\frac{1}{4} b_{1} S_{c}^{2}+\frac{1}{6} e_{1} S_{c}^{3}+\frac{1}{6} e_{2} S_{c}\left|S_{c}\right|^{2} \\
& \left.+\frac{1}{8} d_{1} S_{c}^{4}+\frac{1}{8} d_{3} S_{c}^{2}\left|S_{c}\right|^{2}+\text { H.c. }\right) \\
& +\frac{1}{4} d_{2}\left(\left|S_{c}\right|^{2}\right)^{2}+\frac{\delta_{2}}{2} \Phi^{\dagger} \Phi\left|S_{c}\right|^{2}+\frac{1}{2} b_{2}\left|S_{c}\right|^{2},
\end{aligned}
$$

where $a_{1}, b_{1}, e_{1}, e_{2}, d_{1}, d_{3}, \delta_{1}$ and $\delta_{3}$ are complex. After spontaneous symmetry breaking, in unitary gauge,

$\Phi=\left(\begin{array}{c}0 \\ \frac{h+v}{\sqrt{2}}\end{array}\right), \quad S_{c}=\frac{1}{\sqrt{2}}\left(S+v_{S}+i\left(A+v_{A}\right)\right)$.

Since we have included all allowed terms in Eq. (1), the coefficients can always be redefined such that $v_{S}=$ $v_{A}=0$. This makes the potential of Eq. (1) identical to that obtained by adding 2 real singlets to the SM and there is no $C P$ violation. Previous work $[21,23]$ imposed a global $U(1)$ symmetry or a $Z_{2}$ symmetry to eliminate some of the terms in the potential, making the shift to $v_{S}=v_{A}=0$ in general not possible.

The mass eigenstate fields are $h_{1}, h_{2}, h_{3}$ (masses $m_{1}, m_{2}$, $m_{3}$ ) are found from the rotation,

$$
\left(\begin{array}{l}
h_{1} \\
h_{2} \\
h_{3}
\end{array}\right)=V\left(\begin{array}{l}
h \\
S \\
A
\end{array}\right)
$$

where $V$ is a $3 \times 3$ unitary matrix with,

$$
V \equiv\left(\begin{array}{ccc}
c_{1} & -s_{1} c_{3} & -s_{1} s_{3} \\
s_{1} c_{2} & c_{1} c_{2} c_{3}-s_{2} s_{3} & c_{1} c_{2} s_{3}+s_{2} c_{3} \\
s_{1} s_{2} & c_{1} s_{2} c_{3}+c_{2} s_{3} & c_{1} s_{2} s_{3}-c_{2} c_{3}
\end{array}\right)
$$

and we abbreviate $c_{i}=\cos \theta_{i}$, etc. Note that the phase usually associated with the Cabibbo-Kobayashi-Maskawa (CKM)-like mixing matrix does not appear since the mass matrix in terms of the real fields $h, S$, and $A$ is strictly real by hermiticity. Since all allowed terms are included in Eq. (1), we are free to perform a field redefinition $S_{c} \rightarrow$ $S_{c} e^{i \phi}$ while leaving the form of the potential unchanged. We choose to take $S_{c} \rightarrow S_{c} e^{i \theta_{3}}$. This results in the field redefinitions,

$$
\left(\begin{array}{l}
h \\
S \\
A
\end{array}\right) \rightarrow\left(\begin{array}{ccc}
1 & 0 & 0 \\
0 & c_{3} & -s_{3} \\
0 & s_{3} & c_{3}
\end{array}\right)\left(\begin{array}{l}
h \\
S \\
A
\end{array}\right)
$$

which, when combined with Eqs. (3) and (4) with matrix multiplication, leads to a simplified mixing matrix,

$$
V \rightarrow\left(\begin{array}{ccc}
c_{1} & -s_{1} & 0 \\
s_{1} c_{2} & c_{1} c_{2} & s_{2} \\
s_{1} s_{2} & c_{1} s_{2} & -c_{2}
\end{array}\right)
$$

So we see that performing a suitable phase rotation is equivalent to setting $\theta_{3}=0$. For the rest of the paper, we use this convention to eliminate $\theta_{3}$.

We take as inputs to our scans,

$$
\begin{aligned}
v= & 246 \mathrm{GeV}, \quad m_{1}=125 \mathrm{GeV}, \\
& m_{2}, m_{3}, \theta_{1}, \theta_{2}, \delta_{2}, \delta_{3}, d_{1}, d_{2}, d_{3}, e_{1}, e_{2}
\end{aligned}
$$

where $\delta_{3}, d_{1}, d_{3}, e_{1}$ and $e_{2}$ can be complex and are defined in Eq. (1).

The SM-like Higgs boson is identified with $h_{1}$ with $m_{1}=125 \mathrm{GeV}$. The couplings of $h_{1}$ to SM particles are suppressed by a factor $c_{1}$ relative to the SM rate. The states are ordered according to their couplings to SM particles. $h_{1}$ has the strongest couplings to SM particles, $h_{2}$ couplings are suppressed by $s_{1} c_{2}$ relative to the SM couplings, and $h_{3}$ couplings are the smallest, and are suppressed by $s_{1} s_{2}$ relative to SM couplings. The mass ordering of $h_{2}$ and $h_{3}$ is arbitrary. The ATLAS experiment restricts the value of $c_{1}$ to be,

$$
c_{1}=\left|V_{11}\right|>0.94,
$$

at $95 \%$ confidence level using Run-1 Higgs coupling fits [24]. Similarly, a global fit to Higgs coupling strengths by CMS and ATLAS [25],

$$
\mu=1.09 \pm .11,
$$

yields an identical limit on $c_{1}$.

\section{LIMITS FROM PERTURBATIVITY, OBLIQUE PARAMETERS AND UNITARITY}

The parameters of the model must satisfy constraints from electroweak precision measurements, searches for heavy Higgs bosons, and limits from perturbative unitarity, along with the restrictions from single Higgs production discussed in the previous section. Fits to the oblique parameters place strong limits on the allowed scalar masses and mixings. Analytic results for a model with 2 additional scalar singlets are given in Ref. [26]. For $m_{i} \gg M_{W}, M_{Z}$, the approximate contributions are, 
Limits from Oblique Parameters

$\mathrm{V}_{11}=\cos \theta_{1}=0.94$

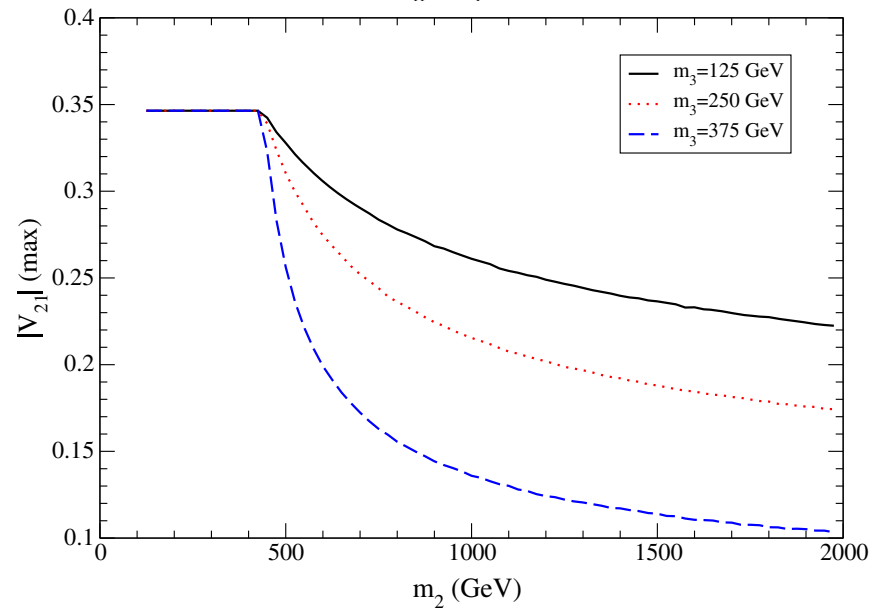

Limits from Oblique Parameters

$\mathrm{V}_{11}=\cos \theta_{1}=0.96$

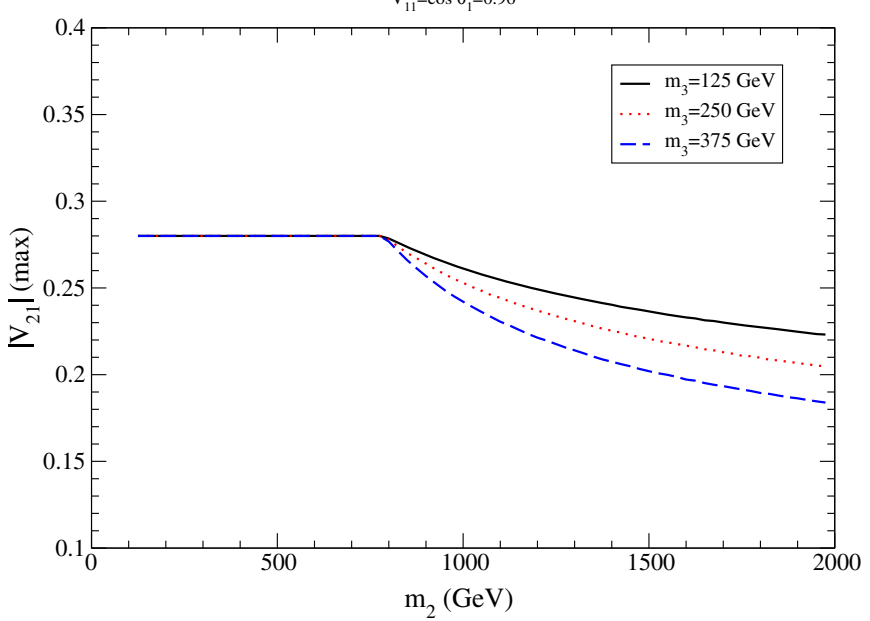

FIG. 1. Limits on $m_{2}$ for allowed couplings of $h_{1}$ to SM particles[ $\cos \theta_{1}=.94$ (LHS) and $\cos \theta_{1}=.96$ (RHS)] for various values of $m_{3}$ using the oblique parameter $(\mathcal{S}, \mathcal{T}, \mathcal{U})$ limits of Ref. [27].

$$
\begin{aligned}
& \Delta \mathcal{S} \sim\left(1-\left|V_{11}\right|^{2}\right) \mathcal{S}_{\mathrm{SM}}+\frac{1}{12 \pi} \Sigma_{i=1,2,3}\left|V_{i 1}\right|^{2} \log \left(\frac{m_{i}^{2}}{m_{1}^{2}}\right) \\
& \Delta \mathcal{T} \sim\left(1-\left|V_{11}\right|^{2}\right) \mathcal{T}_{\mathrm{SM}}-\frac{3}{16 \pi c_{W}^{2}} \Sigma_{i=1,2,3}\left|V_{i 1}\right|^{2} \log \left(\frac{m_{i}^{2}}{m_{1}^{2}}\right) \\
& \Delta \mathcal{U} \sim\left(1-\left|V_{11}\right|^{2}\right) \mathcal{U}_{\mathrm{SM}} .
\end{aligned}
$$

The restrictions from the oblique parameters [27] on $V_{21}=$ $s_{1} c_{2}$ for the minimum value of $c_{1}=.94$ allowed by single Higgs production are shown on the left-hand side (LHS) and for $c_{1}=.96$ on the right-hand side (RHS) of Fig. 1. $\mathrm{TeV}$ scale masses require quite small values of $V_{21}$, which is the parameter that determines the coupling of $h_{2}$ to SM particles. The flat portions of the curves for small $m_{2}$ in Fig. 1 represent the imposed limit on $\theta_{1}$ from single Higgs production. As this limit becomes stronger, the limits from oblique parameters becomes less and less relevant. As the $h_{1}$ couplings become more and more SM-like $\left(\theta_{1} \rightarrow 0\right)$, the allowed coupling of $h_{2}$ to SM particles becomes highly suppressed. The constraints from the oblique parameters shown in Fig. 1 are consistent with those obtained in the real singlet model in Ref. [10]. For the values of $\theta_{2}$ allowed by Fig. 1, the direct searches, $p p \rightarrow h_{2}\left(h_{3}\right) \rightarrow W^{+} W^{-}$do not provide additional restrictions on $V_{21}[28,29]$.

In the real singlet model, much stronger constraints are placed on the parameters from the $W$ boson mass than from the oblique parameters [30,31]. For example, in the real singlet model for $m_{2}=1 \mathrm{TeV}$, the $W$ mass measurement requires $\left|V_{21}\right|<.19$. For $\theta_{2}=0, h_{3}$ does not couple to SM particles and the results of Refs. [30,31] can be applied directly to the complex singlet case. The results of Ref. [30] are shown in Fig. 2. The calculation of the limit from the $W$ mass in the complex singlet model for nonzero $\theta_{2}$ is beyond the scope of this paper and involves contributions from all 3 Higgs bosons and could potentially yield interesting limits. The limits from the oblique parameters in the complex singlet case, (Fig. 1), demonstrates that the dependence of the limits on $m_{3}$ is nontrivial.

The quartic couplings in the potential are strongly limited by the requirement of perturbative unitarity of the $2 \rightarrow 2$ scattering processes [32]. We compute the $J=0$ partial waves, $a_{0}$, in the high energy limit where only the quartic couplings contribute and require $\left|a_{0}\right|<\frac{1}{2}$. The contributions from the trilinear couplings are suppressed at high energy and do not contribute in this limit. For example, we find the restriction from the process, $(S S) / \sqrt{2} \rightarrow(S S) / \sqrt{2}$,

$$
\operatorname{Re}\left(d_{1}+d_{2}+d_{3}\right) \lesssim \frac{32 \pi}{3} .
$$

Similarly, from $h S \rightarrow h S$, we find,

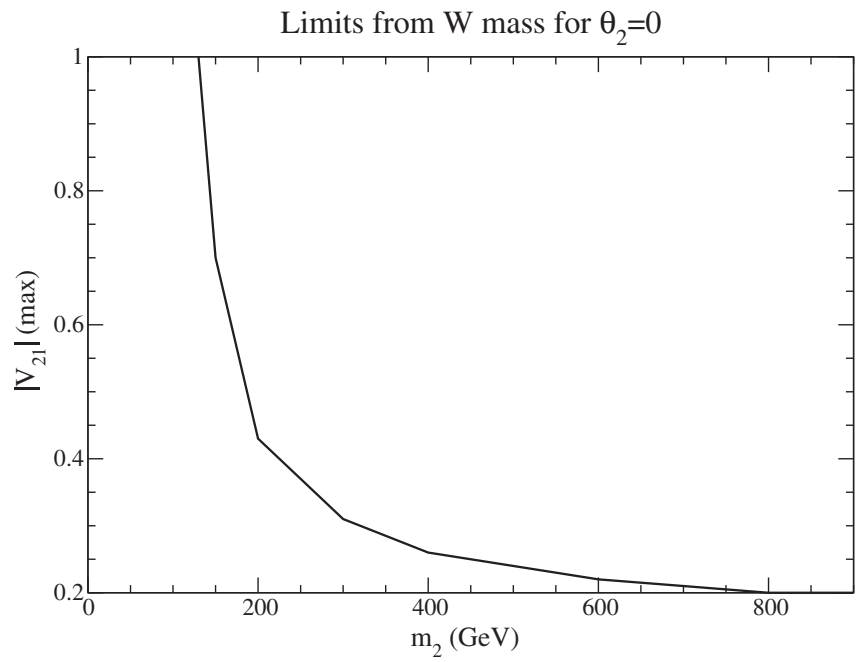

FIG. 2. Maximum allowed value of $V_{21}$ from the $W$ mass measurement as a function of $m_{2}$ in the real singlet model and in the complex singlet model with $\theta_{2}=0$ from Ref. [30]. 


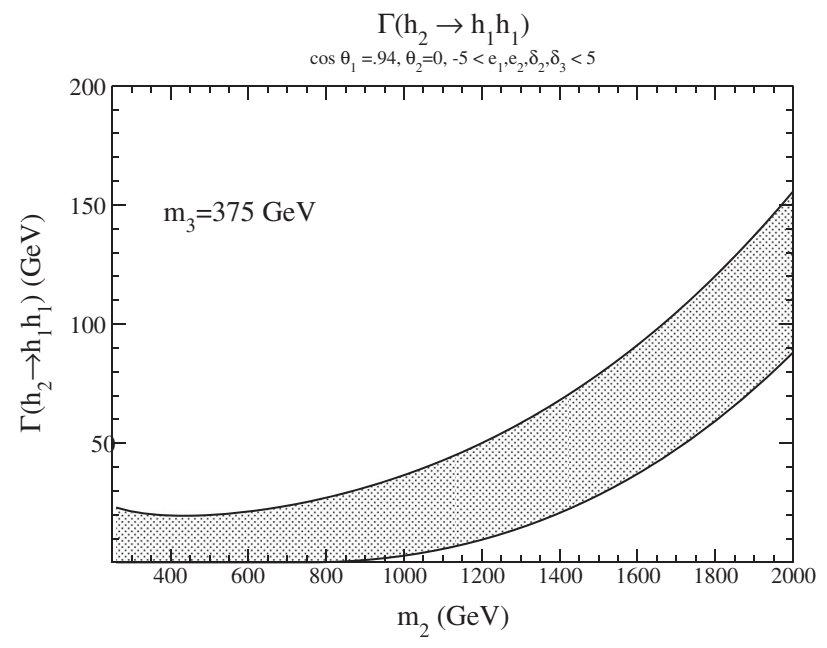

FIG. 3. Decay width for $h_{2} \rightarrow h_{1} h_{1}$ when all parameters are taken real and $\delta_{2}$, and $\delta_{3}$ are scanned over.

$$
\operatorname{Re}\left(\delta_{2}+\delta_{3}\right) \lesssim 16 \pi
$$

Looking at the eigenvectors for neutral $C P$ even scattering processes,

$$
\left\{\omega^{+} \omega^{-}, \frac{z z}{\sqrt{2}}, \frac{h h}{\sqrt{2}}, h S, h A, \frac{S S}{\sqrt{2}}, \frac{A A}{\sqrt{2}}, A S\right\},
$$

$\left(\omega^{ \pm}, z\right.$ are the Goldstone bosons), we find the generic upper limits on the real and imaginary quartic couplings,

$$
\begin{aligned}
\operatorname{Re}\left(d_{i}\right), \operatorname{Im}\left(d_{i}\right) & \lesssim \frac{32 \pi}{3}, \quad i=1,2,3 \\
\delta_{2}, \operatorname{Re}\left(\delta_{3}\right), \operatorname{Im}\left(\delta_{3}\right) & \lesssim 16 \pi .
\end{aligned}
$$

These upper limits are conservative bounds, and more stringent bounds are obtained from looking at the eigenvalues of the 8 by 8 scattering matrix. These upper bounds on the parameters involve finding solutions to higher order polynomials and do not have simple analytic solutions. Thus, the bounds from perturbative unitarity are determined numerically and imposed in the scans of the next section.

The trilinear Higgs couplings depend on the scalar masses and could potentially become large. In the limit of small mixing, $\theta_{1} \ll 1$ and $\theta_{2}=0$, the $h_{2} h_{1} h_{1}$ coupling is,

$\lambda_{211} \rightarrow \sin \theta_{1}\left\{\frac{2 m_{1}^{2}}{v}\left(1+\frac{m_{2}^{2}}{2 m_{1}^{2}}\right)-v\left(\delta_{2}+\operatorname{Re}\left(\delta_{3}\right)\right)\right\}$,

small angle limit

and we see that the growth of $\lambda_{211}$ with large $m_{2}$ is mitigated by the $\sin \left(\theta_{1}\right)$ suppression. The decay width for $h_{2} \rightarrow h_{1} h_{1}$ is [11],

$$
\Gamma\left(h_{2} \rightarrow h_{1} h_{1}\right)=\frac{\lambda_{211}^{2}}{32 \pi m_{2}} \sqrt{1-\frac{4 m_{1}^{2}}{m_{2}^{2}}} .
$$

In Fig. 3, we have taken all parameters real and scanned over $-5<\delta_{2}, \delta_{3}<5$ for fixed $m_{3}, \theta_{1}$ and $\theta_{2}$. The dependence on $e_{1}$ and $e_{2}$ is minimal in the small angle limit, as demonstrated in Eq. (15). In all cases, we have $\Gamma\left(h_{2} \rightarrow h_{1} h_{1}\right) \ll m_{2}$, showing that there is no problem with the trilinear couplings becoming nonperturbative in the small angle limit. Increasing the range we scan over changes the numerical results, but $\Gamma\left(h_{2} \rightarrow h_{1} h_{1}\right) / m_{2}$ is always $\ll 1$.

Finally, we require that the parameters correspond to an absolute minimum of the potential. This has been extensively studied for the real singlet model in Refs. [13,15,19] and analytic results derived. For the case of the complex singlet, we scan over parameter space for numerically allowed values of the parameters [20] and do not obtain an analytic solution.

\section{RESULTS}

In the limit of $\theta_{2} \rightarrow 0$, (as suggested by the single Higgs rates), the scalar $h_{3}$ does not couple directly to SM particles and it can only be observed through di-Higgs production. We will consider $h_{3}$ to be in the $100-400 \mathrm{GeV}$ mass range. The largest production rate at the LHC is through the resonant process $g g \rightarrow h_{2} \rightarrow h_{1} h_{3}$. The complex singlet model is thus an example of new physics that will first be seen in the study of di-Higgs resonances [22,33]. We perform a scan over the parameters of Eq. (7), subject to the restrictions discussed in the previous section. ${ }^{1}$ We always fix $c_{1}=0.94$ and consider the 2 cases, $\theta_{2}=0$ and $\theta_{2}=\frac{\pi}{12}$.

For the allowed parameter space, we compute the amplitude for $g g \rightarrow h_{1} h_{3}$ shown in Fig. 4. Analytic results in the context of the MSSM are given in Ref. [1]. We use the central NLO Les Houches Accord Parton Distribution Functions (LHAPDF) set [34,35], with $\mu_{R}=\mu_{F}=M_{h h}{ }^{2}$ In Fig. 5, we show the invariant $M_{h h}$ spectrum for resonant $h_{1} h_{3}$ production compared to the SM $h_{1} h_{1}$ spectrum at $13 \mathrm{TeV}$. The complex singlet model curves are more sharply peaked than those of the SM and demonstrate a significant enhancement of the rate relative to the SM double Higgs rate for the parameters we have chosen. The spectrum has only a small dependence on $\theta_{2}$, visible at high $M_{h h}$. We have included a finite width for $m_{2}$ in the calculation: For $m_{2}=400 \mathrm{GeV}$ and $m_{3}=130 \mathrm{GeV}$, the width is quite large, $\Gamma_{2}=$ $263 \mathrm{GeV}(\theta=0)$ and $\Gamma_{2}=295 \mathrm{GeV}(\theta=\pi / 12) .^{3}$ We have included the width using the Breit-Wigner approximation, although typically $\Gamma_{2} / m_{2} \sim \mathcal{O}\left(\frac{1}{2}\right)$. The shoulder due to the width is clear on the LHS of Fig. 4. There is a smaller

\footnotetext{
${ }^{1}$ For the complex singlet model with a $U(1)$ symmetry, a comparable scan can be performed using the program SCANNERS [19].

${ }^{2} M_{h h} \equiv\left(p_{h_{1}}+p_{h_{3}}\right)^{2}$

${ }^{3}$ The parameters of the $\theta_{2}=0$ curve on the LHS of Fig. 4 are, for example, $\delta_{2}=21.6, \operatorname{Re}\left(\delta_{3}\right)=-14.5, \operatorname{Im}\left(\delta_{3}\right)=-22.9$, $\operatorname{Re}\left(d_{1}\right)=1.15, \quad \operatorname{Im}\left(d_{1}\right)=1.64, \quad d_{2}=13.3, \quad \operatorname{Re}\left(d_{3}\right)=10.5$, $\operatorname{Im}\left(d_{3}\right)=10.2, \operatorname{Re}\left(e_{1}\right)=1.18 v, \operatorname{Im}\left(e_{1}\right)=-2.66 v, \operatorname{Re}\left(e_{2}\right)=$ $-8.29 v, \operatorname{Im}\left(e_{2}\right)=3.67 v$. These parameters correspond to $\lambda_{211}=$ $-2.9 v \lambda_{311}=6.77 v, \lambda_{321}=-11.1 v$ and $\lambda_{331}=11.2 v$.
} 

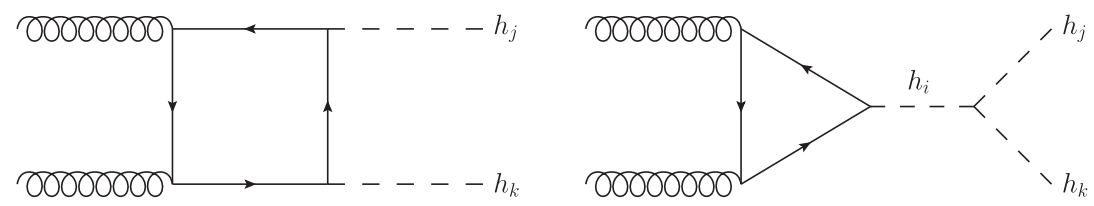

FIG. 4. Feynman diagrams for the production of $h_{j} h_{k}, i, j, k=1,2,3$.
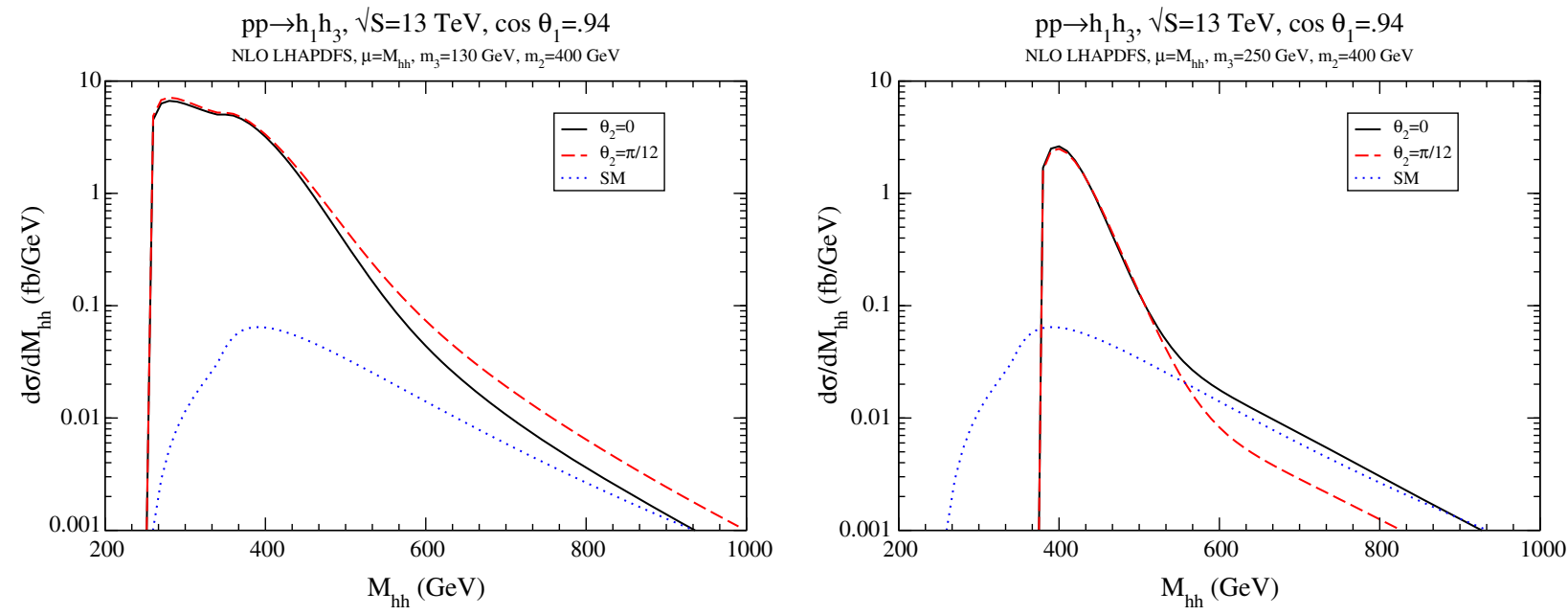

FIG. 5. $M_{h h}$ spectrum of the complex singlet model production of $h_{1} h_{3}$ from the resonant exchange of $h_{2}$. The dominant contribution in the loops is from the top quark.
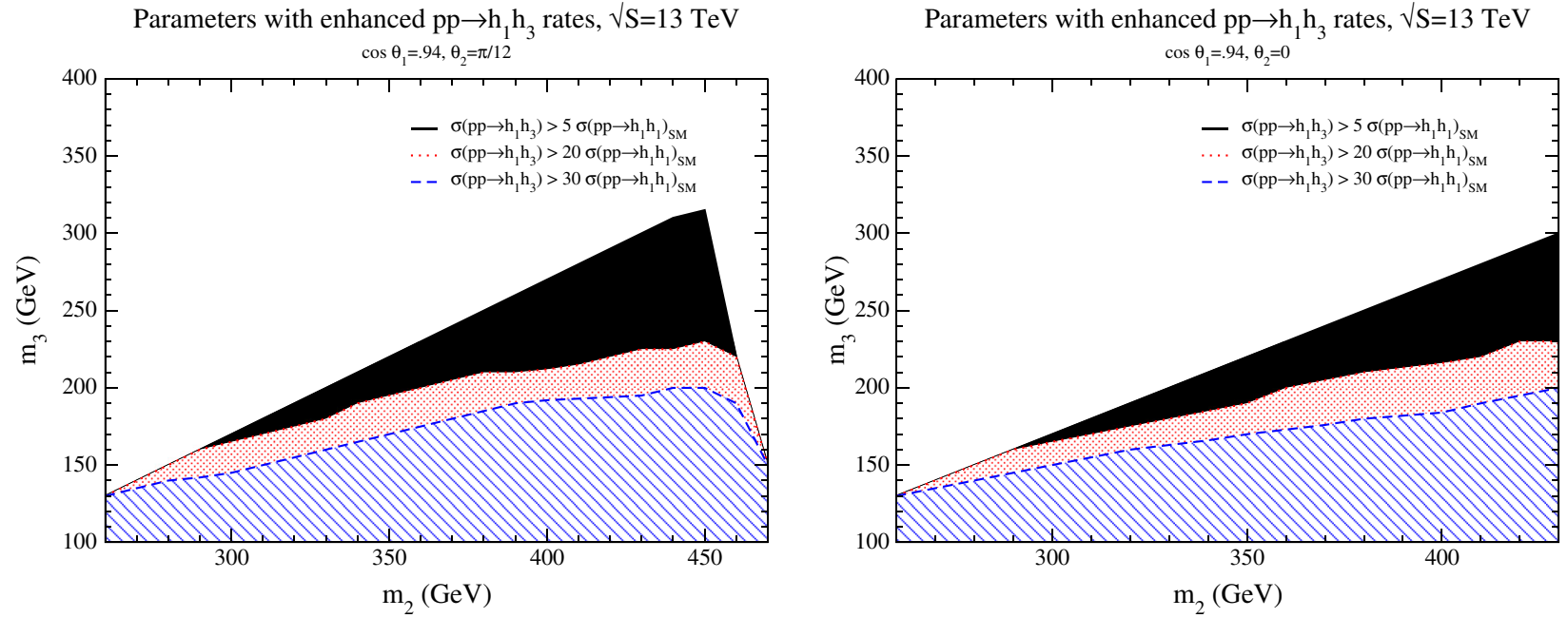

FIG. 6. Regions of parameter space allowed by limits on oblique parameters, perturbative unitarity, and the minimization of the potential where the rate for $h_{1} h_{3}$ production is significantly larger than the SM $h_{1} h_{1}$ rate at $\sqrt{S}=13 \mathrm{TeV}$.

width for $h_{2}$ when $m_{3}$ is increased to $250 \mathrm{GeV}: \Gamma_{2}=$ $129 \operatorname{GeV}(\theta=0)$ and $\Gamma_{2}=137 \operatorname{GeV}(\theta=\pi / 12)$ on the RHS of Fig. 4. The widths are calculated by scaling the SM results from Ref. [36] with the appropriate mixing angles and adding the relevant widths $h_{i} \rightarrow h_{j} h_{k}$.

In Figs. 6 and 7, we show mass regions where the rate for $h_{1} h_{3}$ production is significantly enhanced relative to the $\mathrm{SM} h_{1} h_{1}$ production. This enhancement can be traced to the relatively large values of the trilinear Higgs couplings defined from Eq. (1),

$$
\begin{aligned}
\mathcal{V} & \rightarrow \frac{1}{2} \lambda_{211} h_{1}^{2} h_{2}+\frac{1}{2} \lambda_{311} h_{1}^{2} h_{3}+\frac{1}{2} \lambda_{331} h_{1} h_{3}^{2} \\
& +\lambda_{321} h_{1} h_{2} h_{3}+\cdots,
\end{aligned}
$$



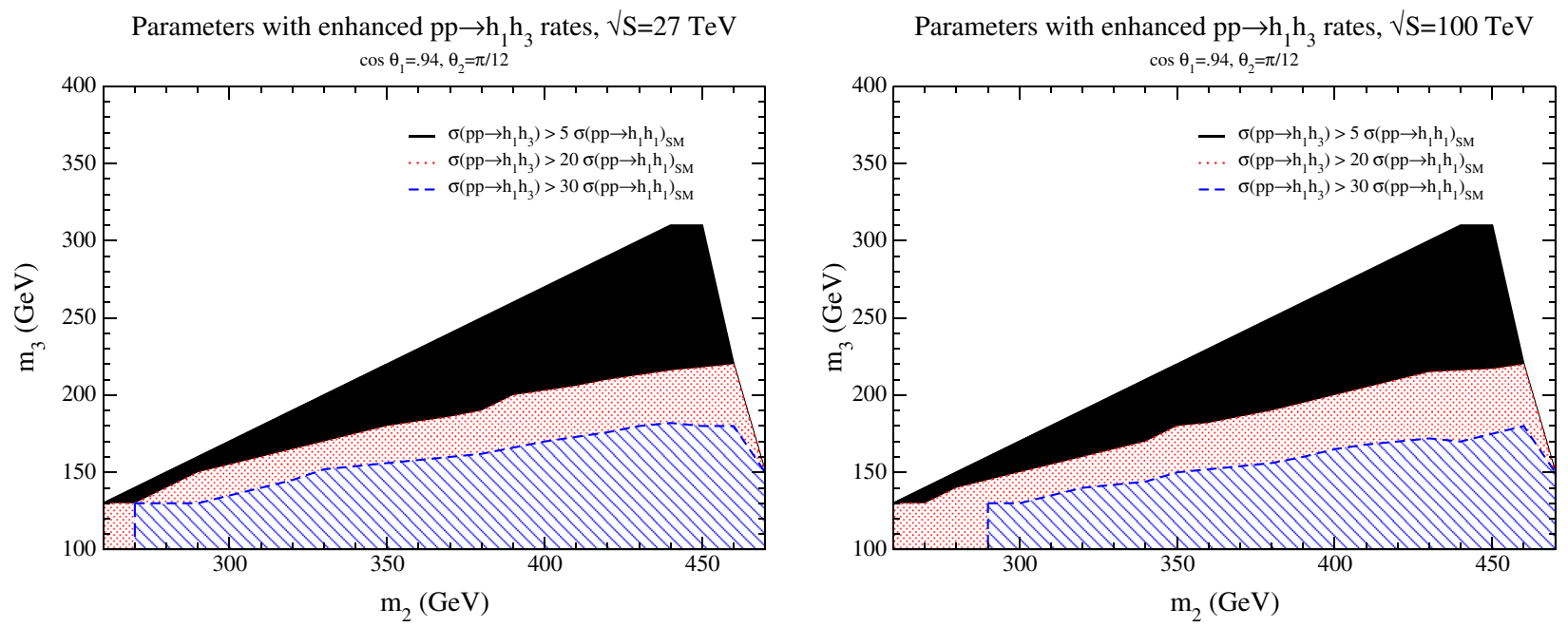

FIG. 7. Regions of parameter space allowed by limits on oblique parameters, perturbative unitarity, and the minimization of the potential where the rate for $h_{1} h_{3}$ production is significantly larger than the $\mathrm{SM} h_{1} h_{1}$ rate at $\sqrt{S}=27 \mathrm{TeV}$ and $100 \mathrm{TeV}$.

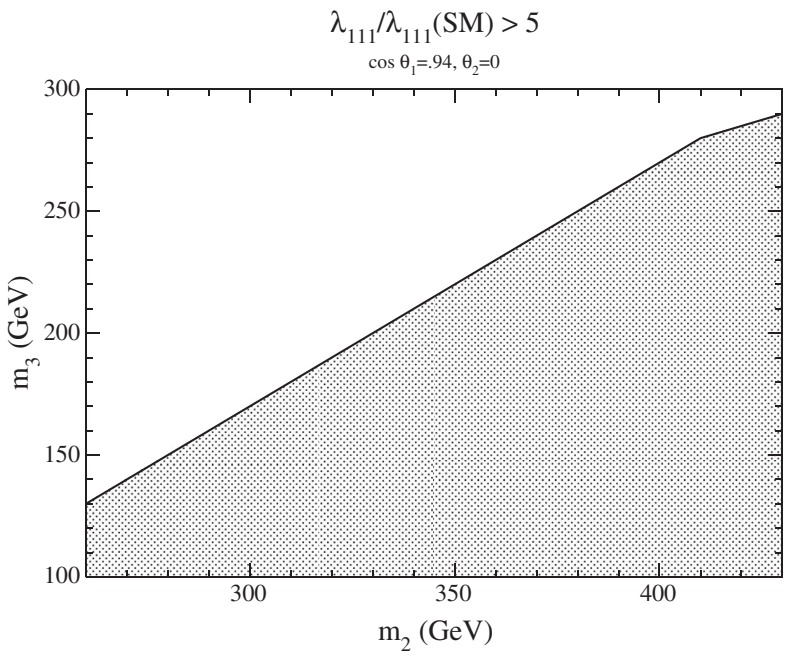

FIG. 8. Region of parameter space allowed by limits on oblique parameters, perturbative unitarity, and the minimization of the potential where the $h_{1} h_{1} h_{1}$ trilinear coupling is greater than 5 times the SM value.

that are allowed by the imposed restrictions. In the SM, the $h h h$ coupling is fixed by $m_{h}$, whereas here, the trilinear couplings of the potential are relatively unconstrained. In Fig. 8, we show the region of parameter space allowed by limits on the oblique parameters, perturbative unitarity, and the minimization of the potential where the $h_{1} h_{1} h_{1}$ trilinear coupling is greater than 5 times the $\mathrm{SM}$ value. This enhancement of the trilinear scalar coupling requires rather light values of $m_{2}$ and $m_{3}$ as shown in Fig. 8. In roughly the same region as shaded in Fig. 8, the $h_{2} h_{1} h_{1}$ and $h_{3} h_{2} h_{1}$ couplings are 8 times the SM $h_{1} h_{1} h_{1}$ coupling. This enhancement is consistent with the results of Ref. [21] in the complex singlet model with a global $U(1)$ symmetry imposed on the potential. The cutoffs on the high $m_{2}$ ends of the plots on the LHS in Figs. 6 and 7 are due to the oblique parameter restrictions in the nonzero $\theta_{2}$ mixing scenario. The same results for $\sqrt{S}=27$ and $100 \mathrm{TeV}$ are shown in Fig. 7. At all energies there is a significant region of phase space where the $h_{1} h_{3}$ rate is large, relative to SM double Higgs production.

For $m_{3}>250 \mathrm{GeV}$, the dominant decay chain from $h_{1} h_{3}$ production will be $h_{1} h_{3} \rightarrow h_{1} h_{1} h_{1} \rightarrow(b \bar{b})(b \bar{b})(b \bar{b})$. For $m_{3}<2 m_{1}, h_{3}$ will decay through the extremely small couplings to SM particles and through the off-shell decay $h_{3} \rightarrow h_{1} h_{1}^{*} \rightarrow h_{1} f \bar{f}$ and will be extremely long lived. In the limiting case where $\theta_{2}=0$, the only allowed decay for $h_{3}$ is the off-shell decay chain through the couplings to $h_{1}$.

\section{CONCLUSIONS}

We have studied an extension of the SM with a complex scalar singlet. We considered the most general renormalizable scalar potential and imposed no additional symmetries. In this scenario, there are 3 scalar bosons, one of which, $h_{3}$, has very small couplings to SM particles and will be primarily observed through di-Higgs decays, $h_{2} \rightarrow h_{1} h_{3}$. Subject to the constraints of electroweak precision measurements, single Higgs production rates, and perturbative unitarity, there are regions of phase space where the rate for $h_{1} h_{3}$ production is significantly enhanced relative to the SM $h_{1} h_{1}$ rate. Therefore, the search for pair production of Higgs bosons with different masses is a distinctive signature of this class of model.

\section{ACKNOWLEDGMENTS}

S. D. is supported by the U.S. Department of Energy under Grant No. DE-AC02-98CH10886 and Contract 
No. DE-AC02-76SF00515. M. S. is supported by the U.S. Department of Energy, Office of Science, Office of Workforce Development for Teachers and Scientists, Office of Science Graduate Student Research (SCGSR) program. The SCGSR program is administered by the Oak Ridge Institute for Science and Education (ORISE) for the DOE. ORISE is managed by ORAU under Contract No. DE-SC0014664. We thank I. M. Lewis for discussions.
[1] T. Plehn, M. Spira, and P. Zerwas, Nucl. Phys. B479, 46 (1996).

[2] R. Frederix, S. Frixione, V. Hirschi, F. Maltoni, O. Mattelaer, P. Torrielli, E. Vryonidou, and M. Zaro, Phys. Lett. B 732, 142 (2014).

[3] ATLAS Collaboration, CERN Report No. ATLAS-CONF2016-049, 2016 (unpublished).

[4] U. Baur, T. Plehn, and D. L. Rainwater, Phys. Rev. D 67, 033003 (2003).

[5] M. J. Dolan, C. Englert, and M. Spannowsky, J. High Energy Phys. 10 (2012) 112.

[6] J. Baglio, A. Djouadi, R. Gröber, M. M. Mühlleitner, J. Quevillon, and M. Spira, J. High Energy Phys. 04 (2013) 151.

[7] V. Barger, L. L. Everett, C. Jackson, A. Peterson, and G. Shaughnessy, Phys. Rev. Lett. 114, 011801 (2015).

[8] V. Barger, P. Langacker, M. McCaskey, M. J. RamseyMusolf, and G. Shaughnessy, Phys. Rev. D 77, 035005 (2008).

[9] S. Profumo, M. J. Ramsey-Musolf, and G. Shaughnessy, J. High Energy Phys. 08 (2007) 010.

[10] G. M. Pruna and T. Robens, Phys. Rev. D 88, 115012 (2013).

[11] C.-Y. Chen, S. Dawson, and I. M. Lewis, Phys. Rev. D 91, 035015 (2015).

[12] S. Dawson and I. M. Lewis, Phys. Rev. D 92, 094023 (2015).

[13] I. M. Lewis and M. Sullivan, Phys. Rev. D 96, 035037 (2017).

[14] J. M. No and M. Ramsey-Musolf, Phys. Rev. D 89, 095031 (2014).

[15] J. R. Espinosa, T. Konstandin, and F. Riva, Nucl. Phys. B854, 592 (2012).

[16] S. Profumo, M. J. Ramsey-Musolf, C. L. Wainwright, and P. Winslow, Phys. Rev. D 91, 035018 (2015).

[17] D. Curtin, P. Meade, and C.-T. Yu, J. High Energy Phys. 11 (2014) 127.
[18] C.-Y. Chen, J. Kozaczuk, and I. M. Lewis, J. High Energy Phys. 08 (2017) 096.

[19] R. Coimbra, M. O. P. Sampaio, and R. Santos, Eur. Phys. J. C 73, 2428 (2013).

[20] M. Gonderinger, H. Lim, and M. J. Ramsey-Musolf, Phys. Rev. D 86, 043511 (2012).

[21] R. Costa, M. Muhlleitner, M. O. P. Sampaio, and R. Santos, J. High Energy Phys. 06 (2016) 034.

[22] M. Muhlleitner, M. O. P. Sampaio, R. Santos, and J. Wittbrodt, J. High Energy Phys. 08 (2017) 132.

[23] V. Barger, P. Langacker, M. McCaskey, M. Ramsey-Musolf, and G. Shaughnessy, Phys. Rev. D 79, 015018 (2009).

[24] G. Aad et al. (ATLAS Collaboration), J. High Energy Phys. 11 (2015) 206.

[25] G. Aad et al. (ATLAS, CMS Collaborations), J. High Energy Phys. 08 (2016) 045.

[26] S. Dawson and W. Yan, Phys. Rev. D 79, 095002 (2009).

[27] J. de Blas, M. Ciuchini, E. Franco, S. Mishima, M. Pierini, L. Reina, and L. Silvestrini, J. High Energy Phys. 12 (2016) 135.

[28] M. Aaboud et al. (ATLAS Collaboration), Eur. Phys. J. C 78, 24 (2018).

[29] V. Khachatryan et al. (CMS Collaboration), J. High Energy Phys. 10 (2015) 144.

[30] D. Lopez-Val and T. Robens, Phys. Rev. D 90, 114018 (2014).

[31] G. Chalons, D. Lopez-Val, T. Robens, and T. Stefaniak, Proc. Sci., DIS2016 (2016) 113 [arXiv:1606.07793].

[32] B. W. Lee, C. Quigg, and H. B. Thacker, Phys. Rev. D 16, 1519 (1977).

[33] M. Bowen, Y. Cui, and J. D. Wells, J. High Energy Phys. 03 (2007) 036.

[34] J. Butterworth et al., J. Phys. G 43, 023001 (2016).

[35] A. Buckley, J. Ferrando, S. Lloyd, K. Nordström, B. Page, M. Rüfenacht, M. Schönherr, and G. Watt, Eur. Phys. J. C 75, 132 (2015).

[36] S. Dittmaier et al. (LHC Higgs Cross Section Working Group), arXiv:1101.0593. 